\title{
Exploring the Reversible Effects of UV Laser Radiation on the Gene Expression Profiles of Saccharomyces cerevisiae Via Network Analysis
}

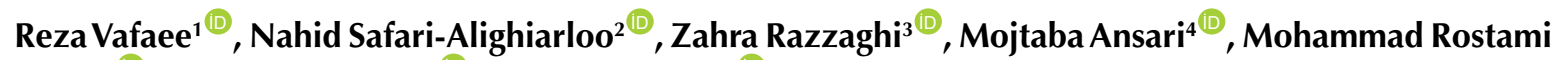 \\ Nejad $^{5}{ }^{\circledR}$, Mostafa Hamdieh $^{6}{ }^{\circledR}$, Vahid Mansouri ${ }^{7 *}$ \\ ${ }^{1}$ Critical Care Quality Improvement Research Center, Faculty of Paramedical Sciences, Shahid Beheshti University \\ of Medical Sciences, Tehran, Iran \\ 2Endocrine Research Center, Institute of Endocrinology and Metabolism, Iran University of Medical Sciences, Tehran, Iran \\ ${ }^{3}$ Laser Application in Medical Sciences Research Center, Shahid Beheshti University of Medical Sciences, Tehran, Iran \\ ${ }^{4}$ Faculty of Medicine, Imam Hosein Hospital, Shahid Beheshti University of Medical Sciences, Tehran, Iran \\ ${ }^{5}$ Research Institute for Gastroenterology and Liver Diseases, Gastroenterology and Liver Diseases Research Center, \\ Shahid Beheshti University of Medical Sciences, Tehran, Iran \\ ${ }^{6}$ Department of Psychosomatic, School of Medicine, Shahid Beheshti University of Medical Sciences, Tehran, Iran \\ ${ }^{7}$ Proteomics Research Center, Faculty of Paramedical Sciences, Shahid Beheshti University of Medical Sciences, Tehran, Iran
}

*Correspondence to

Vahid Mansouri

Email:vm1343@yahoo.com

Received: October 24, 2021 Accepted: December 24, 2021 Published online December 29, 2021

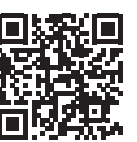

\begin{abstract}
Introduction: The reversibility of biological processes is an important challenge in the study of environmental pollutants and also natural and artificial radiation. There are many pieces of evidence about the reversible and irreversible effects of UV radiation on the human body. Assessment of the reversibility of UV laser effects on Saccharomyces cerevisiae was the aim of this study.

Methods: Gene expression alteration in S. cerevisiae samples radiated by a 30s UV laser for 15 , 30 , and 60 minutes post-radiation times were investigated via network analysis to explore timedependent reversible alteration in the gene expression profiles of the samples.

Results: 19 differentially expressed genes (DEGs) were identified as targeted genes for the samples which were harvested 60 minutes after radiation; network analysis revealed no significant alteration in biological processes.

Conclusion: It can be concluded that the gross effects of the UV laser on S. cerevisiae samples disappear after 60 minutes of radiation.

Keywords: Post-radiation time, Network analysis, Saccharomyces cerevisiae, Repair, Radiation
\end{abstract}

\section{Introduction}

The absorption of UV radiation by cells can affect the structure of DNA and genome. ${ }^{1}$ Damage to nucleic acids and proteins is done either directly by energy absorption or indirectly by the generation of free radicals and single oxygen species. ${ }^{2}$ UV induces cyclobutane pyrimidine dimers production lesions. ${ }^{3}$ A Short wavelength is carcinogenic, and it is absorbed by DNA. Excessive UV radiation causes different skin cancers and damage to the eye lens. ${ }^{5}$ The laser is useful by providing high power, monochromatic, collimated light, and it causes high power to focus on a small area with a local fluence rate. Gasch et al are reported about the transcriptional profile alteration of hundred genes of irradiated cells as an environmental stress response. ${ }^{6}$ Early changes in yeast transcriptome as a suitable biologic model subsequent to a short burst of laser energy were described by Hauser et al. ${ }^{7}$ They mentioned the ability of yeast cells to respond to the UV-induced environmental insult by transcriptional responses which changed the following duration of radiation. However, modifying mechanisms are in place to minimize genome damage. Repairing the excision nucleotide (REN) could remove DNA damage from the transcriptionally silent genome. REN is a complex cellular response to prevent the loss of genetic information in damaged DNA. ${ }^{8}$ For example, in individuals who suffer from Xeroderma pigmentosum as an autosomal recessive disease, UV-induced DNA damages could not be repaired because of the REN defect. ${ }^{9}$ In Saccharomyces cerevisiae yeast as a biological model, short wave radiation causes damage to DNA which stimulate repair process. ${ }^{10} \mathrm{DNA}$ repair mechanisms as a survival factor in this regard is one of the scientists' interests. ${ }^{11}$ The aim of this study was to investigate the time-dependent effects of the UV laser on the gene expression profile of $S$. cerevisiae by using the genomic data of a published document analyzed via network assessment. The results of this study can be useful in relation to an increase in knowledge about the 
application of UV radiation in the clinic.

\section{Materials and Methods}

We analyzed the results of a study by Hauser et al about the differentially expressed genes (DEGs) of S. cerevisiae samples which were exposed to a 30s UV laser and harvested 15 and 30 minutes after radiation (accepted papers by Journal of Lasers in Medical Sciences). On the basis of the methods of the paper published by Hauser et al, "The Explore One XP 355-1 UV laser (Spectra-Physics, Santa Clara CA) controlled by L-Win, a LabView-based graphical user interface" was used for the experiment. ${ }^{7}$ More details of methods are described in the mentioned reference. In the present study, the gene expression profiles of the samples which were exposed to the $30 \mathrm{~s}$ UV laser and harvested 60 minutes after radiation were analyzed, and the results were compared with the samples that were assessed previously. For better resolution, the samples that were radiated $30 \mathrm{~s}$ and harvested 15, 30, and 60 minutes after radiation were categorized in three groups: group-1, group-2, and group-3 respectively.

Like the previously analyzed profiles, $P$ value $<0.05$ and ratio change $>2$ were regarded to explore the significant DEGs. The number of significant DEGs of the three analyzed groups was compared. Maximum values of the fold change of the three groups of the samples were equated. The introduced significant DEGs interacted together via the STRING database and Cytoscape software to form a protein-protein interaction (PPI) network. ${ }^{12}$ Due to weak interactions between the queried DEGs, 60 first neighbors (the optimum number of first neighbor genes that imply maximum interactions between queried DEGs) were added to the queried DEGs, and the network was reconstructed via undirected edges. The constructed network was visualized based on degree value and analyzed by "NetworkAnalyzer" plugin of Cytoscape. Similar to the previous two analyzed profiles, mean+2SD (standard deviation) was regarded as the cutoff of degree value to explore the possible hub nodes. ${ }^{13,14}$ The central nodes were the hub nodes that appeared as top nodes based on betweenness centrality, closeness centrality, and stress.

\section{Results}

Data screening revealed that 19 significant DEGs (based on $P$ value $<0.05$ and ratio change $>2$ ) were targeted genes by the UV laser. As it is depicted in Figure 1, the amounts of significant DEGs for groups 1 and 2 are 452 and 329 respectively. The reduction of DEGs (about 27\%) in group 2 relative to group 1 and $94 \%$ in group 3 compare to group 2 , is highlighted in Figure 1.

The alteration of the maximum value of fold change (based on the data from Hauser and colleagues' report ${ }^{7}$ is presented in Figure 2. It is shown that the maximum values of fold change for groups 1-3 are 2193, 350, and 97 respectively, which are corresponded to severe attenuation

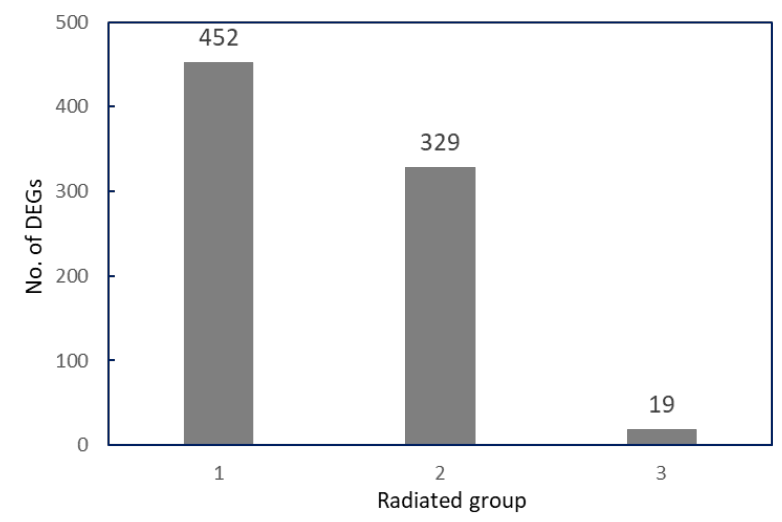

Figure 1. The number of differentially expressed genes for the three studied groups (1-3) that are exposed to a 30s UV laser and harvested 15, 30, and 60 minutes after radiation respectively.

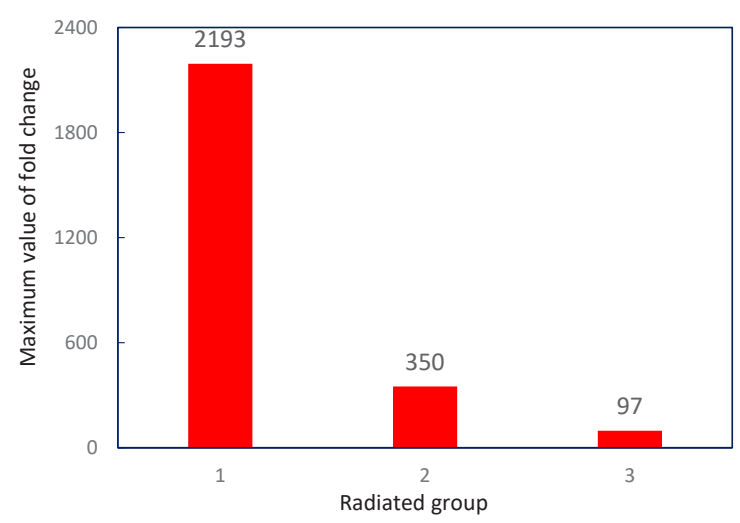

Figure 2. The maximum value of fold change for the groups (1-3) of the samples that are exposed to a 30s UV laser and harvested 15, 30, and 60 minutes after radiation respectively.

correlated with larger post-radiation times.

Interactions between the 19 DEGs are shown in Figure 3. It can be seen that the 19 nodes are connected with 4 edges. However, 12 nodes are isolated, and only 7 genes are connected as three sub-networks. Adding 60 first neighbors leads to constructing a network including 6 isolated nodes and a main connected component which contains 73 nodes (see Figure 4). The number of the central nodes of the networks of the three analyzed groups is presented in Figure 5. The findings indicated that there were 11, 9 and 0 central nodes for groups 1,2 and 3 respectively.

\section{Discussion}

The reversibility or irreversibility of UV radiation is a challenge that has attracted researchers' attention for decades. ${ }^{15,16}$ On the other hand, gene expression profile analysis as a suitable method is used to detect the molecular mechanism of UV radiation. ${ }^{17}$ Here, the reversibility of gene expression alteration due to exposure to the UV laser was assessed via network analysis.

As it is shown in Figure 1, 1-hour post-radiation time attenuated the numbers of significant DEGs from 459 (related to 15-minutes post-radiation time) to 19 . The 


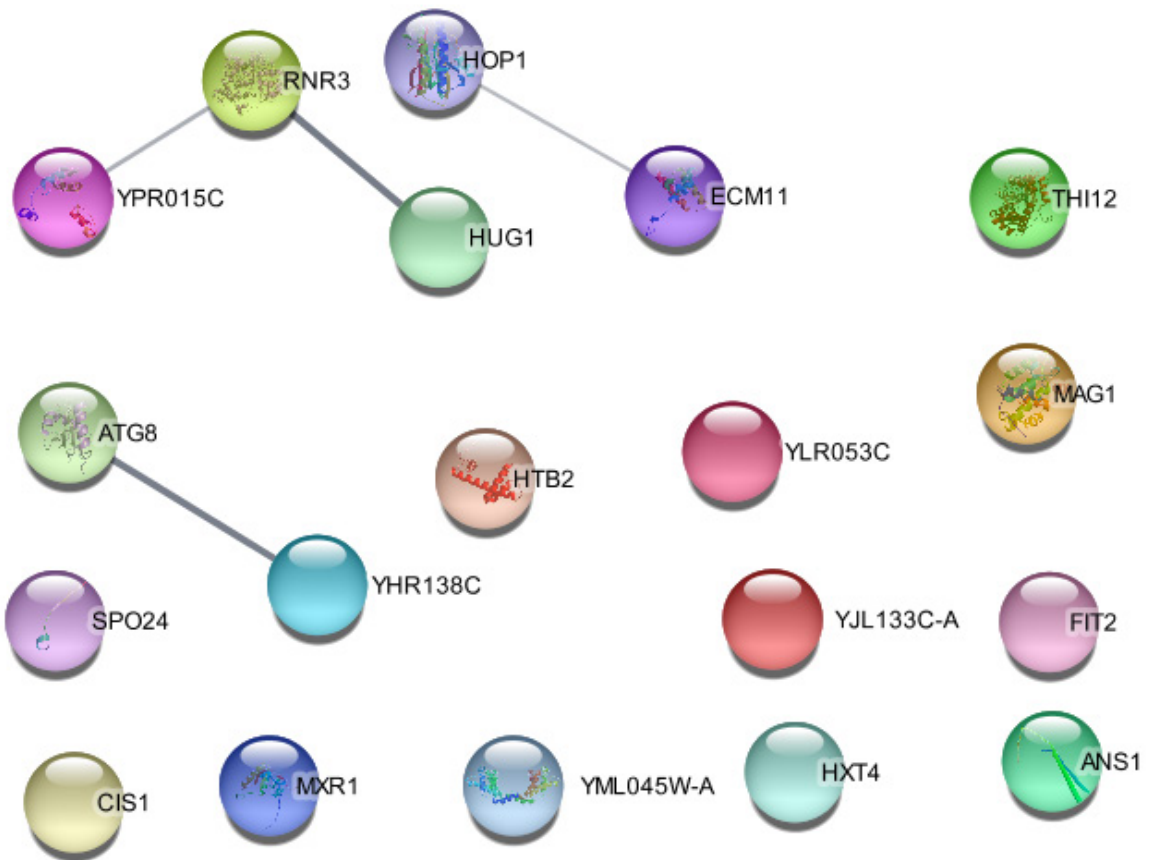

Figure 3. PPI network of the queried DEGs of Saccharomyces cerevisiae samples (group 3) which are exposed to a 30s UV laser and harvested 60 minutes after radiation.

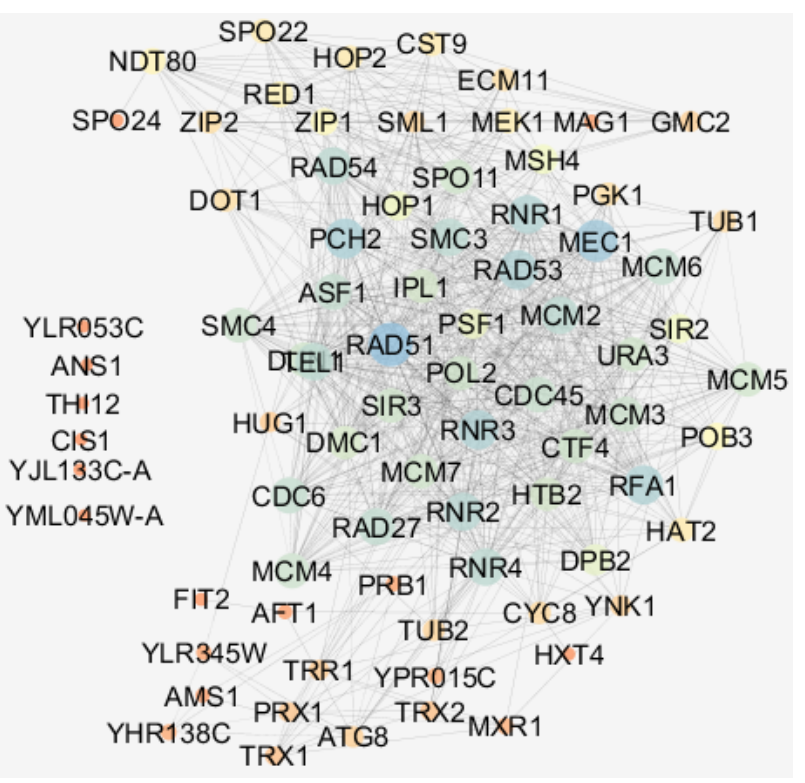

Figure 4. PPI network of the queried DEGs plus the 60 added first neighbors of Saccharomyces cerevisiae samples (group 3) which are exposed to a 30s UV laser and harvested 60 minutes after radiation. Color and size of the nodes are presented based on degree value.

findings indicated that the amounts of the significant DEGs were reduced by about $96 \%$. It may be concluded that $4 \%$ of alterations remained and might be accompanied by biological effects. This idea was real when the range of the maximum value of fold change was reduced by about 96\% (see Figure 2).

Network analysis revealed that the 19 significant DEGs for group-3 cannot interact with each other to form a scale-free network and are almost isolated from each other. As it is shown in Figure 3, there are only 4 edges

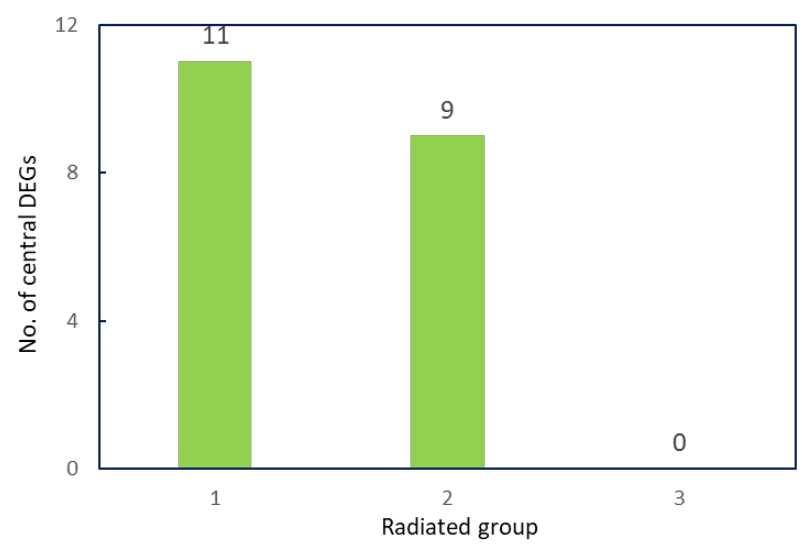

Figure 5. The number of the central genes of the analyzed networks of the studied groups 1-3 that are exposed to a 30s UV laser and harvested 15, 30 , and 60 minutes after radiation.

between the 19 queried DEGs. It seems that network analysis cannot provide useful information about the characters of the queried DEGs.

Adding first neighbors is a useful method to make more interactions between the elements of a network. ${ }^{18}$ Adding 60 first neighbors to the 19 queried DEGs led to constructing a more integrated unit (see Figure 4). It seems that this network contains a valuable finding about the role of the queried significant DEGs. Considering the criteria that the networks of groups 1 and 2 (related to 15 and 30 minutes respectively) were analyzed, network analysis indicated that there were no central nodes for this interactome unit (the network of group-3). As it is represented in Figure 5, numbers of central nodes of the network which was related to group 3 (the group which harvested after 1 hour of radiation) compared to groups 1 
and 2 were reduced to zero.

It has been explored that doubling the time of the yeast cells is approximately 90 minutes $^{19}$; therefore, 60 -minute post-radiation time refers to maximum two generations of the studied yeast cells. There are experiments about the prevention of UV effects on S. cerevisiae by using different agents. Bisquert et al reported the preventive role of melatonin in S. cerevisiae versus UV radiation. ${ }^{20} \mathrm{Li}$ et al have investigated the repair of UV-induced DNA damages in S. cerevisiae. ${ }^{21}$ Guo et al have studied the repair process in $S$. cerevisiae cells that were exposed to X-ray radiation. In this report, it was pointed to the enrichment of DEGs in detoxification and antioxidation activity categories of gene ontology at 1 hour. ${ }^{22}$ Guintini et al studied the repair of telomeres after UV radiation on S. cerevisiae. ${ }^{23}$ We highlighted telomeres as the targeted parts of S. cerevisiae in the previous investigations (In press data).

\section{Conclusion}

The findings indicated that UV laser effects on S. cerevisiae samples were repaired mostly after 1 hour of radiation. More investigation about the repair process in human cell lines such as fibroblast cells is suggested.

\section{Acknowledgment}

Shahid Beheshti University of Medical Sciences supported this research.

\section{Conflict of Interests}

The authors declare they have no conflicts of interest.

\section{Ethical Considerations}

Not applicable.

\section{References}

1. Jan H. HJ Genome maintence mechanisms for preventig cancer. Nature. 2001;411(6835):366-374. doi: $10.1038 / 35077232$

2. Basu AK. DNA damage, mutagenesis and cancer. Int J Mol Sci. 2018;19(4):970. doi:10.3390/ijms19040970

3. Friedberg EC, Walker GC, Siede W, Wood RD, editors. DNA repair and mutagenesis. American Society for Microbiology Press; 2005.

4. de Laat A, van Tilburg M, van der Leun JC, van Vloten WA, de Gruijl FR. Cell cycle kinetics following UVA irradiation in comparison to UVB and UVC irradiation. Photochem Photobiol. 1996;63(4):492-7.

5. Modenese A, Korpinen L, Gobba F. Solar radiation exposure and outdoor work: an underestimated occupational risk. Int J Environ Res Public Health. 2018;15(10):2063. doi:10.3390/ ijerph15102063

6. Gasch AP, Spellman PT, Kao CM, Carmel-Harel O, Eisen $\mathrm{MB}$, Storz G, et al. Genomic expression programs in the response of yeast cells to environmental changes. Mol Biol Cell. 2000;11(12):4241-4257. doi:10.1091/mbc.11.12.4241

7. Hauser M, Abraham PE, Barcelona L, Becker JM. UV laser-induced, time-resolved transcriptome responses of Saccharomyces cerevisiae. G3 (Bethesda). 2019;9(8):25492560. doi:10.1534/g3.119.400291

8. Prakash S, Prakash L. Nucleotide excision repair in yeast. Mutat Res. 2000;451(1-2):13-24. doi:10.1016/s00275107(00)00037-3
9. Gratchev A, Strein P, Utikal J, Goerdt S. Molecular genetics of Xeroderma pigmentosum variant. Exp Dermatol. 2003;12(5):529-536. doi:10.1034/j.1600-0625.2003.00124.x

10. Yin Y, Petes TD. Genome-wide high-resolution mapping of UV-induced mitotic recombination events in Saccharomyces cerevisiae. PLoS Genet. 2013;9(10):e1003894. doi:10.1371/ journal.pgen.1003894

11. Schulz A, Meyer F, Dubrovska A, Borgmann K. Cancer stem cells and radioresistance: DNA repair and beyond. Cancers (Basel). 2019;11(6):862. doi:10.3390/cancers11060862

12. Abbaszadeh H-A, Peyvandi AA, Sadeghi Y, Safaei A, Zamanian-Azodi M, Khoramgah MS, et al. Er:YAG Laser and Cyclosporin A Effect on Cell Cycle Regulation of Human Gingival Fibroblast Cells. J Lasers Med Sci. 2017;8(3):143-149. doi:10.15171/jlms.2017.26

13. Safari-Alighiarloo N, Taghizadeh $M$, Tabatabaei SM, Namaki S, Rezaei-Tavirani M. Identification of common key genes and pathways between type 1 diabetes and multiple sclerosis using transcriptome and interactome analysis. Endocrine. 2020;68(1):81-92. doi:10.1007/s12020019-02181-8

14. Safari-Alighiarloo N, Rezaei-Tavirani $M$, Taghizadeh M, Tabatabaei SM, Namaki S. Network-based analysis of differentially expressed genes in cerebrospinal fluid (CSF) and blood reveals new candidate genes for multiple sclerosis. PeerJ. 2016;4:e2775. doi:10.7717/peerj.2775

15. Lu L, Yang N, Cai Y. Well-controlled reversible additionfragmentation chain transfer radical polymerisation under ultraviolet radiation at ambient temperature. Chem Commun (Camb). 2005;(42):5287-5288. doi:10.1039/ b512057h

16. Sinha R, Häder D-P. Life under solar UV radiation in aquatic organisms. Adv Space Res. 2002;30(6):1547-1556. doi:10.1016/s0273-1177(02)00370-8

17. Yan Y, Stoddard FL, Neugart S, Sadras VO, Lindfors A, Morales LO, et al. Responses of flavonoid profile and associated gene expression to solar blue and UV radiation in two accessions of Vicia faba L. from contrasting UV environments. Photochem Photobiol Sci. 2019;18(2):434-47. doi:10.1039/C8PP00567B

18. Rostami-Nejad M, Razzaghi Z, Esmaeili S, Rezaei-Tavirani $\mathrm{S}$, Baghban AA, Vafaee R. Immunological reactions by $\mathrm{T}$ cell and regulation of crucial genes in treated celiac disease patients. Gastroenterol Hepatol Bed Bench. 2020;13(2):155160.

19. Salari R, Salari R. Investigation of the best Saccharomyces cerevisiae growth condition. Electron Physician. 2017;9(1):3592-3597. doi:10.19082/3592

20. Bisquert R, Muñiz-Calvo S, Guillamón JM. Protective role of intracellular melatonin against oxidative stress and UV radiation in Saccharomyces cerevisiae. Front Microbiol. 2018;9:318. doi:10.3389/fmicb.2018.00318

21. Li W, Adebali O, Yang Y, Selby CP, Sancar A. Proc Natl Acad Sci U S A. 2018;115(15):E3408-E3415. doi:10.1073/ pnas. 1801687115

22. Guo X, Zhang M, Liu R, Gao Y, Yang Y, Li W, et al. Repair characteristics and time-dependent effects in Saccharomyces cerevisiae cells after X-ray irradiation. Appl Microbiol Biotechnol. 2020;104(9):4043-4057. doi:10.1007/ s00253-020-10464-8

23. Guintini L, Tremblay M, Toussaint M, D’Amours A, Wellinger RE, Wellinger RJ, et al. Repair of UV-induced DNA lesions in natural Saccharomyces cerevisiae telomeres is moderated by Sir2 and Sir3, and inhibited by yKu-Sir4 interaction. Nucleic Acids Res. 2017;45(8):4577-4589. doi:10.1093/nar/gkx123 